\title{
LA INFLUENCIA DE LAS REDES SOCIALES VIRTUALES EN LA DIFUS IÓN DE INFORMAC IÓN Y CONOCIMIENTO: ESTUDIO DE PYMES
}

\section{RESUMEN}

El objetivo del trabajo es analizar la influencia de las redes sociales virtuales en la difusión de la información y el conocimiento. La metodología de la investigación se bas a en el estudio de casos e incluy e una revisión de la literatura, la realización de cuestionarios, y el análisis de redes sociales. Como resultado del análisis de casos se observa un limitado uso de las redes sociales, a saber, LinkedIn es utilizado en forma institucional muy acotada y no contribuye a la difusión de información institucional. En el caso de aquellos que utilizan LinkedIn como herramienta para vinculaciones laborales, no evidencian que la red constituya un canal importante de transferencia y absorción de información, y no reflejan convencimiento con respecto a la contribución de las redes sociales en el éxito futuro de la organización.

Palabras clave: Redes Sociales Virtuales; Difusión de Información; Difusión de Conocimiento; LinkedIn; Facebook.

\section{A INFLUÊNCIA DAS REDES SOCIAIS VIRTUAIS NA DISSEMINAÇÃO DE INFORMAÇÃO E CONHECIMENTO: ESTUDO DAS PMES}

\section{RESUMO}

O objetivo do trabalho é analisar a influência das redes sociais virtuais na divulgação da informação e do conhecimento. A metodologia da pesquisa baseia-se em estudos de caso e inclui uma revisão da literatura, a conclusão dos questionários e a análise das redes sociais. Como resultado da análise de casos, há um uso limitado de redes sociais, ou seja, o LinkedIn é usado em uma forma institucional muito limitada e não contribui para a divulgação de informações institucionais. No caso de quem usa o LinkedIn como uma ferramenta para links de emprego, eles não mostram que a rede constitui um importante canal de transferência e absorção de informações e não reflete a convicção quanto à contribuição das redes sociais para o sucesso futuro da organização.

Palavras-chave: Redes Sociais Virtuais; Divulgação de Informação; Divulgação de Conhecimento; Linkedin, Facebook. 
La Influencia de las Redes Sociales Virtuales en la Difusión de Información y Conocimiento: Estudio de PyMES

\section{THE INFLUENCE OF VIRTUAL SOCIAL NETWORKS IN THE DIFFUS ION OF INFORMATION AND KNOWLEDGE: SME STUDY}

\section{ABSTRACT}

The aim of the work is to analyze the influence of virtual social networks in the dissemination of information and knowledge. The methodology is based on case study rese arch and includes a literature review, the use of questionnaires and social network analysis. The case studies revealed a limited use of social networks, namely LinkedIn is used in institutional form to a little extent and does not contribute to the dissemination of institutional information. In the case of those who use LinkedIn as a tool for work links, they do not evidenced that the network is an important channel of transfer and absorption of information, and it does not reflect conviction regarding the contribution of social networks in the future success of the organization.

Keywords: Virtual Social Networks; Information Diffusion; Knowledge Diffusion; LinkedIn; Facebook.

Marisa Analia Sanchez ${ }^{1}$ Maria Alicia Schmidt ${ }^{2}$ Juana Ines Zuntini ${ }^{3}$

Lucrecia $\mathrm{Obiol}^{4}$

\footnotetext{
${ }^{1}$ Doctora en Ciencias de la Computación por la Universidad Nacional del Sur, Argentina. Profesora de la Universidad Nacional del Sur, Buenos Aires - Argentina. E-mail: mas@uns.edu.ar

2 Magister en Administración en Universidad Nacional del Sur, Argentina. Asistente en Dpto. de Ciencias de la Administración del a Universidad Nacional del Sur, Buenos Aires - Argentina. E-mail: alicia.schmidt@uns.edu.ar

${ }^{3}$ Magister en Administración por la Universidad Nacional del Sur, Argentina. Ayudante de docencia en Dpto. de Ciencias de la Administración, Universidad Nacional del Sur, Buenos Aires - Argentina. E-mail: jzuntini@uns.edu.ar

4 Doctorado en Administración por la Universidad Nacional del Sur, Argentina. Profesora Asociada en Dpto. de Ciencias de la Administración, Universidad Nacional del Sur, Buenos Aires - Argentina. E-mail: lucrecia.obiol@uns.edu.ar
} 


\section{INTRODUCCIÓN}

La adquisición, la integración, el almacenamiento y la colaboración en la difusión del conocimiento constituyen capacidades críticas para sustentar la ventaja competitiva de una organización (Grant, 1996), (Chuang, 2004). La capacidad para transferir conocimiento contribuye al desempeño de las organizaciones (Argote \& Ingram, 2000). Por otra parte, la eficacia del proceso de transferencia varía entre las organizaciones y depende de diferentes características (Darr \& Kurtzberg, 2000).

La comunicación a través de redes sociales virtuales tales como Facebook, LinkedIn, Twitter, y los blogs, entre otras, se ha convertido en uno de los principales medios para estar conectados con amigos, empleados, y colegas. Las redes sociales ofrecen un medio económico, ubicuo y masivo, y han generado muchas expectativas debido al potencial para dar apoyo a diferentes funciones de una organización. Muchas organizaciones implementan plataformas de medios sociales como sistemas de gestión del conocimiento para incrementar el conocimiento compartido (Ellison, Gibbs, \& Weber, 2015). En particular, las redes sociales ayudan en la difusión de información y conocimiento haciéndola más eficiente. De todos modos, tal como observan He y Wang (2016) para las organizaciones resulta un desafío integrar los medios sociales dado que los mismos se caracterizan por resultar complejos, informales y episódicos. Asimismo, algunos autores han estudiado si los beneficios de los vínculos sociales también se pueden generar a partir de las redes virtuales. Si bien Internet ofrece la oportunidad de conectar individuos que de otra forma no se hubieran conectado, Van Alstyne y Brynjolfsson (2005) observan que la tecnología tiene el potencial de fragmentar comunidades. Por ejemplo, los usuarios pueden utilizar filtros avanzados para ubicar a otros individuos con los mismos intereses e interactuar solo con ellos.

Considerando el impacto potencial de las redes sociales en la difusión de información y conocimiento, se plantea el objetivo general del trabajo. El mismo es de tipo exploratorio y pretende analizar la influencia de las redes sociales virtuales como instrumento de difusión de información y conocimiento. Para alcanzar el objetivo propuesto se optó por una metodología de estudio de caso múltiple sobre la utilización de redes sociales en organizaciones que se desempeñan en el área de servicios en la ciudad de Bahía Blanca, Argentina. A efectos de cumplimentar el objetivo se deben resolver algunos desafíos tal como la interpretación de las redes con criterios relevantes para la producción, transferencia y aplicación de conocimiento. Con respecto a esto último, la investigación considera la sinergia entre los sectores académico, gubernamental y productivo que ha sido ampliamente considerada para estudiar los formatos institucionales y sociales para la producción, transferencia y aplicación de conocimiento (Etzkowitz \& Leydesddorff, 1995), (Etzkowitz H. , 2003), (Fuerlinger, Fandl, \& Funke, 2015). Además, considerando que los medios sociales constituyen un mecanismo poderoso para alcanzar y mantener el contacto con un gran número de stakeholders, y de esta forma garantizar un diálogo a muy bajo costo (Manetti \& Bellucci, 2016), se analizan los stakeholders representados en las redes estudiadas.

El trabajo se organiza de acuerdo al detalle que aquí se expone. En la siguiente sección se introducen conceptos básicos sobre el análisis de redes sociales. Además, se describen brevemente las plataformas de redes sociales virtuales. La sección 3 incluye una revisión de la literatura para la intersección de los dominios de estudio de redes sociales, difusión de información y ámbito de trabajo. Se detalla la metodología para la revisión, los resultados y una reflexión sobre el estado del arte en el tema. La sección 4 está dedicada a describir los detalles de la metodología utilizada en el estudio de casos. Luego, en la sección 5 se presentan los resultados y su interpretación. Finalmente, en la sección 6 se sintetizan las conclusiones.

\section{CONCEPTOS PRELIMINARES}

\subsection{Breve introducción al Análisis de Redes Sociales}

El Análisis de Redes Sociales aborda su tratamiento de dos formas diferentes. Una utiliza redes egocéntricas que proveen una vista desde la perspectiva del actor en la red, y la otra conceptualiza la estructura completa de la red (Hanneman \& Riddle, 2005), (Edwards, 2010), (Haythornthwaite, 1996). En el primer caso, los vínculos que los actores individuales mantienen con otros describen su propia red egocéntrica. Describen una foto de un actor típico en un ambiente en particular y muestran cuántos vínculos tiene un actor con otro, qué tipo de vínculos mantienen, y qué tipo de información brindan o reciben de otros actores en la red. Este análisis es útil cuando el tamaño de la población es grande o cuando es difícil definir los límites de la población.

En el segundo caso, las redes socio-céntricas o completas describen los vínculos que todos los miembros de un ambiente mantienen con el resto de los miembros del ambiente. En principio, este enfoque requiere respuestas de todos los miembros de un ambiente sobre el resto de los miembros. Este requerimiento limita el tamaño de las redes que se pueden examinar.

Los datos relacionales que se recopilan en un estudio de redes sociales se representan con grafos. Los actores se corresponden con vértices en el grafo, y las 
relaciones se representan con arcos entre los nodos. En los grafos dirigidos, un arco es un par ordenado, y el par ordenado representa la dirección del arco que vincula dos vértices. En los grafos no-dirigidos, no existe una dirección asociada al arco.

La posición de un nodo dentro de su red permite inferir su importancia en el intercambio de información y conocimiento. Se utilizan diferentes indicadores basados en los vértices. El Grado está dado por la cantidad de arcos que inciden en un vértice. Los vértices que están incluidos en muchos de los caminos más cortos entre otros vértices poseen la Centralidad de Intermediación más alta y tienen la capacidad de conectar dos nodos que de otra forma no se vincularían.

Uno de los indicadores globales del grafo está dado por la densidad del grafo que indica cuán interconectados están los vértices entre sí. Una red con una densidad del $100 \%$ se lograría si todos los nodos estuvieran conectados entre sí. Algunos estudios indican que una estructura poco densa (no redundante) favorece el flujo de información y conocimiento dentro de la red. Por otro lado, la densidad puede ser clave para la colaboración dado que facilita la construcción de confianza.

\subsection{Plataformas de redes sociales}

La era moderna de las redes sociales comenzó con la mejora de la performance de Internet a partir de 1995. En el periodo 2002-2004 aparecieron y se promocionaron Cyworld, Friendster, Plaxo, Reunion.com, Hi5, LinkedIn, MySpace, Orkut, Facebook, y Live Spaces (Skeels \& Grudin, 2009). Facebook comenzó como un sitio orientado a los jóvenes en ambientes universitarios y actualmente es utilizado para compartir fotos o vínculos con amigos o publicar mensajes en la "biografia" de otra persona. Dado la próspera tendencia de la utilización Facebook muchas organizaciones se han convertido en unos de los principales usuarios para sus estrategias de marketing (Narayanan, y otros, 2012). Las empresas pagan post promocionales, utilizan servicios de publicidad o crean una "Fan page" en forma gratuita. Los post de las Fan pages se difunden al público en general y a usuarios que suscriben a una Fan page seleccionando el ícono "Me gusta" en la Fan page de la compañía (Shin, Lee, \& Hall, 2014).

LinkedIn se enfoca en la información profesional y estimula a sus usuarios para que construyan un curriculum vitae abreviado y establezcan conexiones laborales. LinkedIn brinda soporte para la creación de grupos a través de una aplicación y un proceso de aceptación. Los grupos incluyen redes de alumnos, empleados de una empresa, una organización profesional o grupo de interés.

Archambault y Grudin (2012) consideraron las redes Facebook, LinkedIn y Twitter para describir cómo se utilizan y si se consideran útiles para la comunicación organizacional y recuperación de contactos. El estudio se basa en una muestra tomada de empleados de Microsoft e incluye un período de cuatro años desde 2008 al 2011. En 2008, los empleados encuestados tenían un perfil y utilizaban LinkedIn (49\%) y Facebook (46\%). Si bien la tendencia es de mayor utilización para ambas redes, si se observa la utilización diaria, muy pocos reportan utilizar LinkedIn. La red profesional LinkedIn resulta más popular en profesionales en el rango de 25 a 40 años y constituye una agenda que se actualiza sola a medida que los contactos cambian de trabajo (Skeels \& Grudin, 2009).

\section{REVISIÓN DE LA LITERATURA}

En esta investigación el tópico redes sociales para la difusión de información y conocimiento en el ámbito de trabajo, se refiere al rol de las redes sociales en la transferencia de información y conocimiento en contextos de pequeñas y medianas organizaciones. La metodología para abordar la revisión de la literatura se basa en la utilizada por los autores Estevez y Janowski (2013) y comprende los siguientes pasos: definir el marco de referencia de evaluación; identificar dominios y recolección de datos; seleccionar artículos relevantes; documentar los artículos seleccionados; analizar los artículos; y definir el estado del arte. A lo largo de esta sección, se presenta el desarrollo de estos pasos.

\subsection{Dominios y dimensiones}

La Figura 1 ilustra los dominios principales y secundarios que contribuyen al tópico. Dado que la búsqueda bibliográfica se realizará utilizando expresiones en idioma inglés en la base Scopus, para facilitar el seguimiento se utilizan abreviaturas basadas en denominaciones en ese idioma. Los dominios principales están dados "Redes sociales virtuales" (OSN por la expresión en inglés "Online Social Networks"), "Difusión de Información/Conocimiento" (ID por "Information/knowledge diffusion") y "ámbito de trabajo" (W por "Workplace"). Los dominios secundarios corresponden con "redes sociales para la difusión de información" (OSN4ID por "Social networks for Information diffusion") como la intersección de OSN y ID; redes sociales en el ámbito de trabajo(OSN@W por "Online Social networks at the workplace"); y difusión de la información en el ámbito de trabajo (ID@W por "Information diffusion at the workplace").

El alcance de los dominios OSN e ID es amplio. En este trabajo se describe el alcance de los dominios en términos de las dimensiones relevantes al objetivo general del trabajo. Las investigaciones en OSN abarcan múltiples disciplinas. El dominio OSN constituye uno de los ejemplos más notables del denominado "mundo convergente de lo físico y 
cibernético" (traducido del inglés "Cyber-Physical converging World") (Fu, Passarella, Quercia, Sala, \& Strufe, 2016). Desde el punto de vista de la convergencia físico-cibernética el mundo físico y el mundo cibernético ("facilitados" por las tecnologías y servicios de Internet) están interactuando constantemente e influenciando el estado de cada uno (Conti, y otros, 2012). En el caso de OSN, un ejemplo es la sugerencia de amistad: datos sobre individuos en el mundo físico son elaborados para identificar posibles amigos (conocimiento), los cuales son sugeridos a los usuarios que pueden establecer una amistad en la OSN (acción) (Conti, y otros, 2012). Conti et al. destacan algunos de los tópicos de investigación más importantes en el campo OSN y categorizan cada tópico en base a su principal interés en el mundo físico o cibernético. Los tópicos del mundo cibernético incluyen "Privacidad y Confianza", "OSN Descentralizadas", y "Gestión de Contenidos". Existen trabajos que pueden apreciarse en la intersección de los mundos físico y cibernético: "Redes sociales basadas en la ubicación", "Redes sociales móviles", "Propiedades estructurales y relacionales", y "Difusión de la información y análisis de sentimientos". Los autores identifican otro conjunto de tópicos, donde las OSN se consideran como herramientas para investigar la conducta humana social en el mundo real, o como apoyo a las tareas diarias: apoyo a la comunidad, sugerencia de conductas, y microscopio social (es decir, como una herramientas para recolectar datos a gran escala con una evidencia cuantitativa a gran escala basada en propiedades de las redes sociales) (Conti, y otros, 2012). Para el propósito de esta investigación, la clasificación de tópicos representará dimensiones relevantes de OSN.

La transferencia de conocimiento en las organizaciones es el proceso a través del cual una unidad (por ejemplo, un grupo, departamento, o división) es afectado por la experiencia de otro (Argote \& Ingram, 2000). Tal como observan Argote e Ingram (2000) si bien la transferencia de conocimiento en las organizaciones involucra la transferencia a nivel individual, el problema trasciende el nivel individual para incluir niveles superiores tales como grupos, líneas de productos, departamentos o divisiones. La difusión de conocimiento ha sido estudiada en varias disciplinas: psicología social (se refiere a la cognición y aprendizaje social que estudia las comunicaciones y el procesamiento de información en grupos humanos investigando los procesos cognitivos subyacentes); economía y administración (estudios en el conocimiento de innovaciones); ciencia de redes (cómo el conocimiento se difunde en redes sociales y cómo evoluciona la red social) (Luo, Du, Liu, Xuan, \& Wang, 2015). Con respecto al último tópico, las investigaciones exploran la difusión en redes que combinan vínculos internos y externos de la organización. A partir de lo expuesto, surgen las siguientes dimensiones: "Cognición y aprendizaje social", "Conocimiento de innovaciones", "Difusión de información y análisis de sentimientos" y "Apoyo a comunidades". Las últimas dos dimensiones coinciden con aquellas que surgen de las investigaciones en OSNs.

Figura 1 - Dominios de estudio.

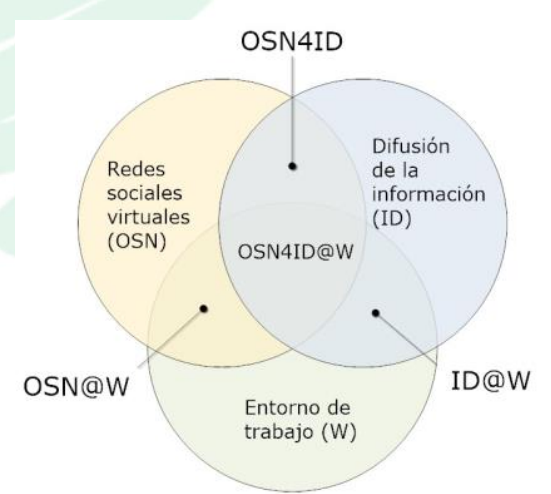

Fuente: elaboración propia.

\subsection{Marco de referencia para la evaluación}

El marco de referencia para la evaluación de artículos se basa en el trabajo de Ahlemann et al. (2013) y se adaptan las definiciones acorde a los objetivos de la presente revisión. Los autores describen la naturaleza de la investigación en la disciplina de la gestión de proyectos. Realizan la revisión y clasificación de la literatura categorizando los trabajos de acuerdo a la naturaleza de la investigación, la cual se codifica con los valores descriptiva, prescriptiva, conceptual, teórica u otra (ver Tabla 1). 
La Influencia de las Redes Sociales Virtuales en la Difusión de Información y Conocimiento: Estudio de PyMES

Tabla 1 - Tipos de investigación.

\begin{tabular}{|c|l|}
\hline Descriptiva & $\begin{array}{l}\text { La investigación descriptiva responde preguntas vinculadas del tipo qué } \\
\text { y cómo así como también del tipo Si/No. }\end{array}$ \\
\hline Prescriptiva & $\begin{array}{l}\text { La investigación prescriptiva busca asistir en la resolución de problemas } \\
\text { prácticos desarrollando y testeando artefactos (por ejemplo, un método, un } \\
\text { modelo, una ontolgía). }\end{array}$ \\
\hline Conceptual & $\begin{array}{l}\text { Los artículos conceptuales presentan supuestos, premisas, axiomas, } \\
\text { afirmaciones, sin un trabajo empírico. }\end{array}$ \\
\hline Teórica & $\begin{array}{l}\text { Una teoría debe satisfacer tres criterios: (1) una teoría debe incluir } \\
\text { constructores claros; (2) las relaciones entre los constructores debe estar } \\
\text { definida; y (3) una teoría debe poder verificarse. }\end{array}$ \\
\hline Otra & $\begin{array}{l}\text { Análisis de la literatura, editoriales, reportes, revisiones de libros, y } \\
\text { convocatorias a trabajos/resúmenes/participaciones. }\end{array}$ \\
\hline
\end{tabular}

Fuente: adaptado de Ahlemann, F., El Arbi, F., Kaiser, M., \& Heck, A. (2013).

La investigación prescriptiva se analiza más ampliamente considerando los siguientes constructores: tipo de resultados, fundamento teórico, métodos utilizados para el desarrollo de la solución, y el método de evaluación. El constructor Tipo de Resultado se refiere a las soluciones elaboradas por una investigación prescriptiva, es decir, un método, un modelo, un marco de referencia, una ontología, un modelo de referencia o un sistema. El constructor Fundamento teórico se refiere al proceso de desarrollo de artefactos para justificar decisiones de diseño. Los valores vinculados a este constructor se recuperan de palabras clave utilizadas en el artículo analizado. El constructor Método de desarrollo de la solución puede asumir los siguientes valores: análisis de la literatura, deducción matemática o lógica, y análisis empírico de datos. El constructor Método de evaluación representa al método utilizado para evaluar la efectividad del artefacto propuesto. Para asegurar que la investigación prescriptiva realmente brinde los beneficios esperados, los investigadores utilizan los siguientes métodos (si es utiliza alguno): estudio de casos, simulación, encuesta, opinión de expertos, meta-análisis, revisión de la literatura, y análisis de textos.

Las categorías de investigación descriptiva, conceptual o teórica se categorizan considerando el tipo de pregunta que el artículo pretende responder.

Figura 2 - Componentes utilizados para el análisis.

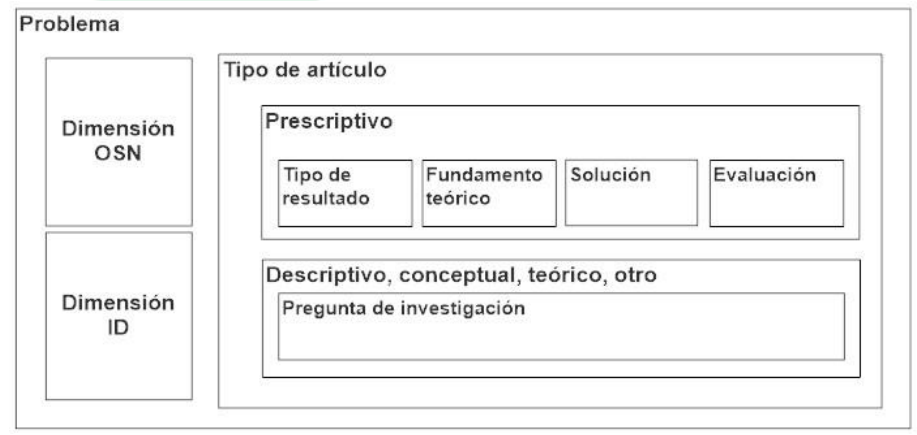

Fuente: elaboración propia.

\subsection{Recolección de datos}

Como se mencionó previamente, se definieron tres dominios primarios - OSN, ID y $\mathrm{W}$, tres dominios secundarios -OSN4ID, OSN@W y ID@W. Dado que el objetivo del presente trabajo es analizar la literatura sobre redes sociales que integra aspectos de difusión de información, solo se incluyen OSN4ID y OSN@W. Con respecto al lugar de trabajo, se consideran ámbitos de pequeñas y medianas organizaciones. Se efectuaron búsquedas en la base de datos Scopus considerando la aparición de un conjunto de palabras en títulos, resúmenes y palabras clave. Para cada dominio se utilizaron las palabras incluidas en la Tabla 2. El análisis se restringe a los últimos seis años (2011 a mediados de enero de 2017), dado que esta ventana temporal debiera ser adecuada para proveer una descripción completa de las investigaciones actuales en redes sociales. 
Tabla 2 - Dominios y palabras clave utilizadas en las búsquedas.

\begin{tabular}{|c|c|}
\hline Dominios & Expresiones utilizadas en Scopus \\
\hline $\begin{array}{l}\text { Redes sociales virtuales para la } \\
\text { difusión de información y } \\
\text { conocimiento (OSN4ID) }\end{array}$ & $\begin{array}{l}\text { "Virtual social networks" AND "Information diffusion", "Virtual } \\
\text { social networks" AND "Information transfer", "Virtual social } \\
\text { networks" AND "Knowledge diffusion", "Virtual social networks" } \\
\text { AND "Knowledge transfer", "Online social networks" AND } \\
\text { "Information diffusion", "Online social networks" AND } \\
\text { "Information transfer", "Online social networks" AND "Knowledge } \\
\text { diffusion", "Online social networks" AND "Knowledge transfer" }\end{array}$ \\
\hline $\begin{array}{l}\text { Redes sociales virtuales en el } \\
\text { lugar de trabajo (OSN@W) }\end{array}$ & $\begin{array}{l}\text { "Virtual social networks" AND "Small and medium size } \\
\text { enterprises", "Online social networks" AND "Small and medium } \\
\text { size enterprises", "Virtual social networks" AND "Workplace", } \\
\text { "Online social networks" AND "Workplace" }\end{array}$ \\
\hline
\end{tabular}

Fuente: elaboración propia

\subsection{Análisis de datos}

Las Tablas 3 y 4 incluyen el número de artículos que se recuperaron en cada búsqueda (un total de 268). La difusión de información ha atraído el interés de la investigación desde diversas áreas, tales como las ciencias de la computación, la física, la biología, las ciencias sociales, entre otras. La mayoría de los trabajos recuperados en Scopus están asociados al área de ciencias de la computación y en esta área los objetivos más destacados responden a (a) qué información o tópicos resultan más populares; (b) cómo y en cuáles caminos de una red se difunde la información, y (c) cuáles miembros de la red desempeñan un papel importante en la difusión de la información. En este trabajo no se pretende modelar el fenómeno de difusión de información sino explorar si las organizaciones utilizan redes sociales, qué tipo de contactos registran y si las perciben como un medio de transferencia y absorción de información y conocimiento. Atendiendo al objetivo de la presente revisión, solo se consideran las revistas que están clasificadas en la base de datos Scopus en las siguientes áreas temáticas: "Ciencias Sociales" (la denominación en inglés en Scopus corresponde con "Social Sciences"), "Negocios, Administración y Contabilidad" ("Business, Management and Accounting"), "Multidisciplinaria" ("Multidisciplinary"), "Economía, econometría y Finanzas" ("Economics, Econometrics and Finance") y "Psicología" ("Psychology"). Con este filtro, resultan 32 de los 268 artículos iniciales. Los problemas OSN4ID@W fueron clasificados de acuerdo a las dimensiones definidas para los dominios OSN e ID (Tabla 5). En algunos casos, más de una dimensión resulta apropiada y la dimensión más cercana a los propósitos de este trabajo fue considerada. Se puede observar que la cantidad de trabajos publicados en el dominio OSN4ID ha crecido desde el año 2011 con un pico en el año 2015. 
La Influencia de las Redes Sociales Virtuales en la Difusión de Información y Conocimiento: Estudio de PyMES

Tabla 3 - Número de artículos para los dominios OSN4ID y OSN@W en la base Scopus.

\begin{tabular}{|c|c|c|c|c|c|c|c|c|}
\hline $\begin{array}{c}\text { Subdominios y expresiones de } \\
\text { búsqueda }\end{array}$ & 2011 & 2012 & 2013 & 2014 & 2015 & 2016 & 2017 & Total \\
\hline \multicolumn{9}{|c|}{ Redes sociales virtuales para la difusión de información y conocimiento (OSN4ID) } \\
\hline $\begin{array}{l}\text { "Virtual social networks" AND } \\
\text { "Information diffusion" }\end{array}$ & - & 1 & - & 2 & - & - & 1 & 4 \\
\hline $\begin{array}{l}\text { "Virtual social networks" AND } \\
\text { "Information transfer" }\end{array}$ & - & - & - & - & - & - & - & 0 \\
\hline $\begin{array}{l}\text { "Virtual social networks" AND } \\
\text { "Knowledge diffusion" }\end{array}$ & - & - & - & - & - & - & - & 0 \\
\hline $\begin{array}{l}\text { "Virtual social networks" AND } \\
\text { "Knowledge transfer" }\end{array}$ & - & - & - & - & - & - & - & O \\
\hline $\begin{array}{l}\text { "Online social networks" AND } \\
\text { "Information diffusion" }\end{array}$ & 15 & 25 & 35 & 37 & 58 & 47 & 1 & 218 \\
\hline $\begin{array}{l}\text { "Online social networks" AND } \\
\text { "Information transfer" }\end{array}$ & 1 & 1 & - & - & - & - & 1 & 3 \\
\hline $\begin{array}{l}\text { "Online social networks" AND } \\
\text { "Knowledge diffusion" }\end{array}$ & - & - & 4 & - & - & - & - & 4 \\
\hline $\begin{array}{l}\text { "Online social networks" AND } \\
\text { "Knowledge transfer" }\end{array}$ & 2 & 6 & 7 & 1 & 1 & 2 & - & 19 \\
\hline \multicolumn{9}{|c|}{ Redes sociales virtuales en el lugar de trabajo (OSN@W) } \\
\hline \begin{tabular}{llr|} 
"Virtual social networks" & AND \\
"Small and medium & size \\
enterprises" & & \\
\end{tabular} & - & - & - & 1 & - & - & - & 1 \\
\hline $\begin{array}{llr}\text { Online social } & \text { networks" } \\
\text { "Small and } & \text { AND } \\
\text { medium } & \text { size } \\
\text { enterprises" } & & \end{array}$ & - & & - & - & 1 & - & - & 1 \\
\hline $\begin{array}{l}\text { "Virtual social networks" AND } \\
\text { "Workplace" }\end{array}$ & - & - & - & - & - & - & - & $\mathbf{0}$ \\
\hline $\begin{array}{l}\text { "Online social networks" AND } \\
\text { "Workplace" }\end{array}$ & 2 & - & 8 & 4 & 3 & 1 & - & 18 \\
\hline Total & 20 & 33 & 52 & 45 & 63 & 51 & 2 & 268 \\
\hline
\end{tabular}

Fuente: elaboración propia a partir de los datos procesados.

Tabla 4 - Tipo de artículos.

\begin{tabular}{|l}
\begin{tabular}{|l|c|c|c|c|c|c|c|}
\hline \multicolumn{1}{|c|}{ Subdominios y expresiones de búsqueda } & Conferencia & $\begin{array}{c}\text { Artículo de } \\
\text { revista }\end{array}$ & Revisión & Capítulo & Libro & Total \\
\hline \begin{tabular}{|l|c|c|c|c|c|} 
"Vedes sociales virtuales para la difusión de información y conocimiento (OSN4ID) \\
"Information diffusion" AND
\end{tabular} & 3 & 1 & - & - & - & $\mathbf{4}$ \\
\hline $\begin{array}{l}\text { "Virtual social networks" AND } \\
\text { "Information transfer" }\end{array}$ & - & - & - & - & - & $\mathbf{0}$ \\
\hline $\begin{array}{l}\text { "Virtual social networks" AND } \\
\text { "Knowledge diffusion" }\end{array}$ & - & - & - & - & - & $\mathbf{0}$ \\
\hline $\begin{array}{l}\text { "Virtual social networks" AND } \\
\text { "Knowledge transfer" }\end{array}$ & 134 & 76 & 4 & 3 & 1 & $\mathbf{2 1 8}$ \\
\hline $\begin{array}{l}\text { "Online social networks" AND } \\
\text { "Information diffusion" }\end{array}$ & 1 & 2 & - & - & - & $\mathbf{3}$ \\
\hline $\begin{array}{l}\text { "Online social networks" AND } \\
\text { "Information transfer" }\end{array}$ & - & - & - & - & $\mathbf{0}$ \\
\hline $\begin{array}{l}\text { "Online social networks" AND "Knowledge } \\
\text { diffusion" }\end{array}$ & 4 & - & - & - & - & $\mathbf{4}$ \\
\hline $\begin{array}{l}\text { "Online social networks" AND "Knowledge } \\
\text { transfer" }\end{array}$ & 15 & 3 & 1 & - & - & $\mathbf{1 9}$ \\
\hline
\end{tabular}
\end{tabular}




\begin{tabular}{|c|c|c|c|c|c|c|}
\hline \multicolumn{7}{|c|}{ Redes sociales virtuales en el lugar de trabajo (OSN@W) } \\
\hline $\begin{array}{l}\text { "Virtual social networks" AND "Small and } \\
\text { medium size enterprises" }\end{array}$ & - & 1 & - & - & - & 1 \\
\hline $\begin{array}{l}\text { "Online social networks" AND "Small and } \\
\text { medium size enterprises" }\end{array}$ & - & 1 & - & - & - & 1 \\
\hline $\begin{array}{l}\text { "Virtual social networks" AND } \\
\text { "Workplace" }\end{array}$ & - & - & - & - & - & $\mathbf{0}$ \\
\hline $\begin{array}{l}\text { "Online social networks" AND } \\
\text { "Workplace" }\end{array}$ & 11 & 7 & - & - & - & 18 \\
\hline Total & 168 & 91 & 5 & 3 & 1 & 268 \\
\hline
\end{tabular}

Fuente: elaboración propia a partir de los datos procesados.

Tabla 5 - Resultados basados en las dimensiones consideradas para los dominios "Redes sociales virtuales" y "Difusión de la información".

\begin{tabular}{|c|c|c|}
\hline Dimensión OSN e ID & Número & Referencias de artículos analizados \\
\hline Sugerencia de conductas & 3 & \multirow{7}{*}{$\begin{array}{l}\text { (Agrawal et al., 2011), (Aragon et al., 2013), (Chelmis et al., 2013), } \\
\text { (Chelmis et al. 2014), (Crowne et al., 2015), (Garg et al., 2011), } \\
\text { (Heimbach \& Hinz, 2016), (Jiang et al., 2014), (Korzynski, 2014), } \\
\text { (Levy et al., 2016), (Li et al., 2016), (Liang et al., 2016), (Lim et al, } \\
\text { 2011), (Lin et al., 2012), (Lin et al., 2014), (Lipizzi et al., 2016), } \\
\text { (Mozafari \& Hamzeh, 2015), (Palacios-Marquez et al., 2016), (Roy } \\
\text { \& Zeng, 2015), (Sankar \& Ravindran, 2015), (Smith, 2013), } \\
\text { (Subires-Mancera \& Olmedo Salar, 2013), (Tang et al., 2015), } \\
\text { (Wadhwa \& Bhatia, 2015), (Wang et al., 2015), (Wu et al., 2015), } \\
\text { (Zaglia et al, 2015), (Zhang, 2015), (Zhao et al., 2011), (Zinoviev, } \\
\text { 2011). }\end{array}$} \\
\hline Soporte a la comunidad & 8 & \\
\hline Difusión de información & 9 & \\
\hline $\begin{array}{l}\text { Conocimiento de } \\
\text { innovaciones }\end{array}$ & 1 & \\
\hline Análisis de sentimientos & 1 & \\
\hline Microscopio social & 9 & \\
\hline $\begin{array}{c}\text { Propiedades estructurales } \\
\text { y relacionales }\end{array}$ & 1 & \\
\hline Total & 32 & \\
\hline
\end{tabular}

Fuente: elaboración propia a partir de los datos procesados.

Las dimensiones "Microscopio social" y "Difusión de información" contribuyen con el $28 \%$ de los artículos cada una. "Soporte a la comunidad" sigue con el $25 \%$. Esto no es sorprendente dado que solo se incluyeron en el análisis detallado artículos provenientes de las áreas "Ciencias Sociales", "Negocios, Administración y Contabilidad", "Multidisciplinaria", "Economía, econometría y Finanzas" y "Psicología". Con respecto al tipo de artículo, 16 (50\%) fueron clasificados como prescriptivos, $9(28,1 \%)$ como descriptivos; $6(18,8 \%)$ como Otras; y una como conceptual. La Tabla 6 resume el análisis de los trabajos prescriptivos. La mayoría definen hipótesis sobre la utilización de las redes sociales virtuales y desarrollan la solución considerando investigaciones relacionadas. Se utilizan datos empíricos para validar las hipótesis y como resultado, se propone un modelo sobre la conducta investigada. Los artículos cuyas contribuciones corresponden con métodos proponen nuevas formas de analizar redes sociales virtuales. El fundamento teórico predominante corresponde con el análisis de redes sociales. 
La Influencia de las Redes Sociales Virtuales en la Difusión de Información y Conocimiento: Estudio de PyMES

Tabla 6 - Artículos correspondientes a la investigación prescriptiva.

\begin{tabular}{|clc|}
\hline Componente analizada & Cantidad \\
\hline \multirow{3}{*}{ Tipo de resultado } & Método & 5 \\
& Modelo & 10 \\
& Otro & 1 \\
\hline & Modelo basado en agentes, Propagación de información & 2 \\
& Teoría de juegos, redes sociales & 1 \\
& Redes sociales implícitas, Difusión de información & 2 \\
& Redes sociales & 3 \\
\multirow{2}{*}{ Fundamento teórico } & Redes sociales, Modelos basados en agentes & 1 \\
& Redes sociales, Proceso de Markov & 1 \\
& Redes sociales, Minería de datos & 3 \\
& Redes sociales, Toma de decisiones grupales & 1 \\
& Redes sociales, Aprendizaje multi-tarea & 1 \\
& Redes sociales, Optimización & 1 \\
\hline Método de desarrollo & Análisis empírico de datos & 1 \\
de la solución & Análisis literario & 15 \\
\hline Evaluación de & Empírica & 15 \\
resultados & No hay evaluación & 1 \\
\hline
\end{tabular}

Fuente: Elaboración propia a partir de los datos procesados.

Con respecto a las investigaciones descriptivas, conceptuales o teóricas, se identifica a la pregunta considerada en el artículo a partir del análisis de su resumen. No se observa que predomine ningún tópico (Tabla 7).

Tabla 7 - Tópicos en las investigaciones conceptuales, descriptivas y de otro tipo.

\begin{tabular}{|lccc|}
\hline & \multicolumn{3}{c|}{ Tipo de investigación } \\
\cline { 2 - 4 } & Conceptual & Descriptiva & Otra \\
\hline Redes sociales virtuales basadas en conferencias & 0 & 1 & 0 \\
Viralidad de contenidos & 0 & 1 & 0 \\
Recursos humanos & 0 & 0 & 1 \\
Difusión de información & 0 & 1 & 0 \\
Predicción de vínculos & 0 & 1 & 0 \\
Campañas políticas en línea & 0 & 1 & 0 \\
Productividad & 1 & 1 & 0 \\
Revisiones & 0 & 0 & 3 \\
Rol de redes sociales virtuales en la innovación & 0 & 1 & 0 \\
Señales multimedios sociales & 0 & 0 & 1 \\
Adopción de tecnología en el ámbito de trabajo & 0 & 2 & 0 \\
Analítica visual & 0 & 0 & 1 \\
\hline
\end{tabular}

Fuente: elaboración propia a partir de los datos procesados.

Como se desprende de la categorización efectuada en el relevamiento de artículos académicos seleccionados, el análisis subyacente sugiere una naturaleza de investigación predominante de tipo prescriptivo. En contraposición a esto, se destaca un escaso desarrollo de trabajos de investigación conceptual y ninguna teorización.

Un análisis más profundo de los trabajos más cercanos a los objetivos de la investigación destaca las siguientes investigaciones. Zaglia et al. (2015) examinan cómo los gerentes de pequeñas y medianas empresas perciben a las redes sociales virtuales como beneficiosa para sus empresas. Como resultado de una encuesta que suministran a propietarios y gerentes de empresas y usuarios de la plataforma XING, los autores concluyen que la confianza y el acceso a recursos sustentan la satisfacción con la red. Los miembros están satisfechos si el valor ganado de la red supera el tiempo dedicado. La participación en la red tiene un impacto en el desempeño percibido de la empresa dado que provee acceso a recursos y capacidades. En la misma línea de investigación, Palacios-Marqués et al. (2016) exploran el efecto de las redes sociales y la gestión basada en competencias en 
la capacidad de innovación. Se realiza una encuesta en empresas españolas de biotecnología y telecomunicaciones la cual confirma que la utilización de la redes para lectura, búsqueda y almacenamiento de información, y para compartir y co-crear conocimiento afecta positivamente la transferencia del conocimiento y este conocimiento ayuda a que las empresas mejoren las competencias en investigación y desarrollo.

Zhang et al. (2016) abordan el problema de estudiar la difusión de información entre empleados en base a contactos virtuales y tradicionales (personales, telefónicos). Proponen un modelo que indica cómo extraer canales de difusión, cómo procesar la información distribuida por los diferentes canales y cómo ponderar y seleccionar los canales. La validación del modelo la realizan a partir de consideran información de empleados de Microsoft registrados en la red social Yammer, todos los contenidos generados por estos empleados en la red (posteos, respuestas) y relaciones que emergen de relaciones tales como seguimiento a usuarios o tópicos en la red, o membrecías a grupos. Una limitación práctica de la propuesta resulta de la disponibilidad de los datos necesarios para aplicar el modelo. Subires Mancera y Olmedo Solar (2013) pretenden conocer y valorar la predisposición de docentes, estudiantes y profesionales al uso de redes sociales de carácter académico profesional. Los autores realizan una encuesta la cual revela que las redes generalistas como Facebook, Youtube y Twitter lideran las preferencias, y el primer objetivo de uso de las redes es contactar con amigos y luego establecer contacto con profesionales del mismo campo de trabajo. Si bien se observa una predisposición a participar de una red, no se refleja una evidencia de que se esté utilizando efectivamente para facilitar el trabajo colaborativo.

\section{METOdología PARA EL ANÁLISIS DE CASOS}

El método utilizado es el estudio de casos múltiples (Yin, 1994). La unidad de análisis es la organización en la cual el contacto trabaja (que contestó el cuestionario y brindó información sobre su red profesional). Para cada organización se recopiló información a partir de contactos profesionales registrados en la red LinkedIn; la actividad en la Fan Page de la organización (si existe) y un cuestionario. El cuestionario permite indagar sobre as pectos difíciles de inferir a partir de las redes virtuales. De esta forma se pretende cotejar las características de la organización, las características relacionales de la red social virtual y la percepción que tienen los contactos sobre el rol de la red en el intercambio de información y conocimiento. A continuación, se brindan más detalles metodológicos sobre cómo se procede para realizar el análisis de los datos provenientes de LinkedIn, Facebook y el cuestionarios sobre propiedades relacionales.

\subsection{Red profesional LinkedIn}

Se propone construir una red que surge de la red de contactos profesionales LinkedIn de un contacto que se desempeña en la organización considerada. En el análisis de la red se calculará la densidad del grafo para tener un indicador de cuán conectados están los nodos de la red entre sí. Además, para cada nodo se indicará el grado y la centralidad de intermediación. No se desarrollan otros indicadores dado que cada red está construida a partir de una sola persona (ego), por lo tanto, los valores de otros indicadores no agregan información.

A partir de la información pública que cada miembro describe en LinkedIn se clasificará a cada miembro de acuerdo a la pertenencia al ámbito académico, gubernamental o productivo; y el país de residencia. La clasificación en estos sectores pretende analizar la capacidad de la red para difundir información entre diferentes sectores y explorar la sinergia entre los mismos. Se basa en el modelo de la Triple Hélice. El modelo de la Triple Hélice resalta la importancia de la sinergia entre los sectores académico, gubernamental y productivo con el objetivo de generar nuevos formatos institucionales y sociales para la producción, transferencia y aplicación de conocimiento (Etzkowitz \& Leydesddorff, 1995), (Etzkowitz H. , 2003), (Fuerlinger, Fandl, \& Funke, 2015). Dado que algunos usuarios de las redes sociales reflejan un desempeño balanceado en dos sectores (académico y productivo), se optó por clasificarlo como "A-P" para denotar su potencial influencia en los sectores académico y productivo.

Además, con el objetivo de analizar las interacciones entre diferentes actividades económicas, se clasifican los nodos de la red considerando la función que desempeñan actualmente y publicada en su perfil de la red social. La clasificación se realiza de acuerdo a la Clasificación Industrial Internacional Uniforme de todas las actividades económicas (CIIU) (Naciones Unidas, 2009). La misma constituye la clasificación internacional de las actividades productivas y aporta cuatro niveles de clasificación (secciones, divisiones, grupos y clases). Luego de realizar un análisis preliminar de los datos, se observó que para muy pocos casos se dispone de datos para determinar la clase. Por lo tanto, a efectos de elaborar clasificaciones comparables se utiliza el nivel de división para caracterizar a los nodos de la red.

Los actores de la red profesional también se clasifican desde la perspectiva de stakeholders. Algunos autores han analizado a los medios sociales basados en Internet como herramienta para fortalecer el compromiso de los stakeholders. Manetti et al. (Manetti \& Bellucci, 2016) evalúan si la interacción a través de Facebook, Twitter y Yotube representa un mecanismo efectivo de compromiso de los stakeholders para definir los contenidos de un reporte social, ambiental o de sustentabilidad. Se concluye que un pequeño número de organizaciones utilizan los 
medios sociales para el objetivo enunciado y que el nivel de interacción mayoritariamente es bajo.

\subsection{Interacciones en la Fan Page de Facebook}

El análisis de la Fan Page resulta un indicador de la importancia que asigna la organización a la gestión de redes sociales. Por ejemplo, la frecuencia de actualización y el tiempo de respuesta a consultas reflejan este aspecto. A partir de cada Fan Page de interés se construye una red en la cual los nodos corresponden con usuarios y se conectan los usuarios que indicaron "Me gusta" o comentaron sobre el mismo post. Debido a que los usuarios de Facebook muchas veces utilizan un nombre de fantasía y por lo tanto, no resulta factible efectuar una clasificación del perfil.

\subsection{Características relacionales}

Para facilitar la interpretación de las redes virtuales y complementar el análisis con propiedades relacionales difíciles de inferir a partir de la estructura de las redes sociales virtuales, se instrumentó cuestionario. El instrumento es un cuestionario estructurado. El mismo se envió a los contactos que brindaron información sobre la red LinkedIn.

Se formularon preguntas para caracterizar el flujo de información y conocimiento adapatadas de la propuesta de Fritsch y Kauffeld-Monz (2010). Los autores analizan la transferencia de información y conocimiento en una muestra de redes de innovación.

La fuerza de los lazos involucra una combinación de cantidad de tiempo, servicios recíprocos, servicios recíprocos e intensidad emocional (Granovetter, 1973). Dada una red, es difícil cuantificar para cada contacto su antigüedad o la frecuencia de comunicación. Dado que se desea estudiar la red en el contexto de la difusión de información y conocimiento, la cantidad de tiempo se aproximó con la frecuencia en que el individuo se enriquece con información, sugerencias o estímulos que sus contactos realizan en la red (se utilizó una escala de Likert de 5 puntos). Inspirado en el trabajo de Gilsing (2008), los servicios recíprocos se aproximan con el alcance de los contenidos y se indaga sobre los temas que el individuo se informa a través de la red. La Tabla 8 describe el resto de las características relacionales evaluadas. El cuestionario incluye otras preguntas para caracterizar el tipo de actividad; la antigüedad de la organización; el tamaño (cantidad de empleados); mercado geográfico (local, regional, nacional, internacional); desempeño general de la organización en los últimos tres años (participación en el mercado, ventas, utilidad sobre ventas, productividad, retorno sobre la inversión). Además, se indaga sobre el número de membrecías en diferentes tipos de redes (Cámara de comercio, Asociaciones industriales o sectoriales, Grupos de interés, Grupos virtuales de interés). La diversidad de membrecías en diferentes redes brinda diferentes puentes con grupos heterogéneos.

Tabla 8 - Resumen de variables e indicadores.

\begin{tabular}{|c|c|}
\hline Flujo de la información & Indicador \\
\hline $\begin{array}{c}\begin{array}{c}\text { Absorción de } \\
\text { información }\end{array} \\
\text { Flujo de conocimiento }\end{array}$ & $\begin{array}{l}\text { Pregunta: "En su opinión, ¿sus contactos de LinkedIn se benefician de la } \\
\text { información o sugerencias que Usted realiza en dicha red social?". Escala de } \\
\text { Likert de } 5 \text { puntos ("Muy poco" = } 1 \text {; "Mucho" }=5 \text { ). } \\
\text { Pregunta: "Usted, ¿se enriquece de la información, sugerencias o estímulos } \\
\text { que realizan sus contactos en LinkedIn?". Escala de Likert de } 5 \text { puntos } \\
\text { ("Muy poco"=1; "Mucho"=5). }\end{array}$ \\
\hline $\begin{array}{l}\text { Transferencia de } \\
\text { conocimiento } \\
\text { Absorción de } \\
\text { conocimiento } \\
\text { Fuerza de la relación }\end{array}$ & $\begin{array}{l}\text { Pregunta: Sus contactos ¿se benefician de la asistencia técnica y/o } \\
\text { profesional que Usted le brinda en la red Linkedin?". Escala de Likert de } 5 \\
\text { puntos ("Muy poco"=1; "Mucho" }=5 \text { ). } \\
\text { Pregunta: "Usted ¿se beneficia con la asistencia técnica y/o profesional que } \\
\text { recibe desde la red Linkedin?". Escala de Likert de } 5 \text { puntos ("Muy poco" = } \\
\text { 1; "Mucho"=5). }\end{array}$ \\
\hline Cantidad de tiempo & $\begin{array}{l}\text { Frecuencia de acceso a la red. Escala de Likert de } 5 \text { puntos (Nunca, Rara } \\
\text { vez, A veces, A menudo, Siempre). } \\
\text { Pregunta: "¿Usted diría que gracias a la red se beneficia de la siguiente } \\
\text { información?". Selección múltiple de temas (Conocimiento científico; } \\
\text { Información técnica y recursos; Know-how de administración/legales; } \\
\text { Información de mercado; Recursos financieros; Nunca por ningún motivo; } \\
\text { No sabe). } \\
\text { Pregunta: "Usted diría que entre los contactos existe honestidad y } \\
\text { confianza?". Escala de Likert de } 5 \text { puntos con las categorías "De ninguna } \\
\text { manera" }=1 \text { y "Mucha" }=5 \text {. }\end{array}$ \\
\hline
\end{tabular}


Intensidad emocional

Pregunta: “¿En su organización se considera que las redes sociales (grupos de interés en Facebook, contactos o grupos en LinkedIn) que facilitan la colaboración y el conocimiento mutuo con otras organizaciones contribuyen en el éxito futuro de la organización?". Escala de Likert de 5 puntos ("En desacuerdo" = 1; "Totalmente de acuerdo" =5).

Fuente: elaboración propia.

\section{RESULTADOS DEL ESTUDIO DE CASOS}

Se analizaron tres empresas que brindan servicios, a saber: servicios de consultoría informática, servicios de seguros en el área de la salud, y servicios de ingeniería. La Tabla 9 resume características de las organizaciones. A continuación, se presentan los resultados para cada caso.

Tabla 9 - Características de las organizaciones consideradas.

\begin{tabular}{|c|c|c|c|c|c|c|c|c|}
\hline \multirow[b]{2}{*}{ 률류 } & \multirow[b]{2}{*}{ 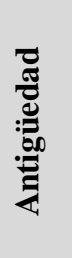 } & \multirow[b]{2}{*}{ 胥 } & \multirow[b]{2}{*}{ 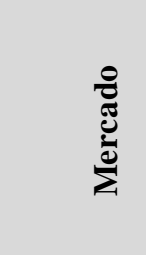 } & \multicolumn{5}{|c|}{ Presencia en redes sociales } \\
\hline & & & & 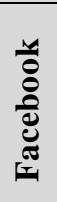 & 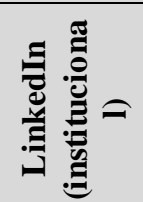 & 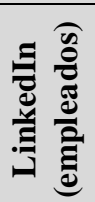 & 赵 & $\underset{\partial}{\stackrel{\Xi}{E}}$ \\
\hline \multicolumn{9}{|c|}{ Zuntini Computación } \\
\hline 62 & 40 & Menos de 9 & Regional & $\mathrm{Si}$ & $\mathrm{Si}$ & - & - & $\mathrm{Si}$ \\
\hline \multicolumn{9}{|c|}{ Prevención Salud } \\
\hline 65 & 20 & Más de 100 & Nacional & $\mathrm{Si}$ & - & $\mathrm{Si}$ & $\mathrm{Si}$ & $\mathrm{Si}$ \\
\hline \multicolumn{9}{|c|}{ AF Equipamientos } \\
\hline 71 & 15 & Menos de 9 & Nacional & - & - & $\mathrm{Si}$ & - & - \\
\hline
\end{tabular}

Referencias: *Código según la Clasificación Industrial Internacional Uniforme de todas las actividades económicas (CIIU) (Naciones Unidas, 2009).

Fuente: elaboración propia.

\subsection{Zuntini Computación}

\section{Descripción de la organización}

Se trata de una empresa fundada en 1974 que inició sus actividades con la venta de máquinas de escribir y computadoras XT. Desde hace más de 20 años incorporó la venta de los Controladores Fiscales, llegando a ser grandes referentes en las provincias argentinas de Buenos Aires, La Pampa y Río Negro. Su misión es apoyar el desarrollo de factores competitivos estratégicos de los clientes, ofreciendo soluciones integrales mediante la transferencia de tecnología de informática, y brindar un constante asesoramiento integral en las áreas requeridas por los clientes. La empresa se focaliza en la tecnología de avanzada y las soluciones integrales, ofreciendo las mejores marcas, y servicio técnico pos-venta. En relación a los competidores y rivales de la organización existen pocas firmas de fuerte presencia y, por sobre todo, con larga permanencia en el mercado. Los clientes se vinculan con áreas muy variadas que van desde la agropecuaria, al de las corporaciones, cooperativas eléctricas, reparticiones públicas, consorcio de puertos, y público en general. Asimismo, se trabaja en contacto con empresas locales y nacionales, sobre todo el sector de PyMEs.

Sitio web. La organización cuenta con un sitio web actualizado y con referencias a la Fan Page de Facebook, un canal Youtube y a "Google Mi Negocio" con información actualizada de la organización.

Facebook. Se realizó un análisis preliminar de la Fan Page para determinar la fecha de inicio, la cantidad de seguidores y la frecuencia de la actividad (publicación de posts). A partir de los grafos que representan las redes se computó los datos incluidos en la Tabla 10. Para el periodo analizado se observa una publicación semanal de posts los cuales se refieren a consejos informáticos.

LinkedIn. Con respecto a los contactos profesionales se definió una red y se calcularon las métricas basadas en el grafo: cantidad de vértices (100), $\operatorname{arcos}(402)$ y densidad $(0,08)$ (ver Figura 3 y Tabla 11). Como la red está generada a partir de un contacto, el máximo valor para el grado corresponde con ese contacto. La densidad de los grupos que surgen a partir de los sectores o stakeholders no resulta superior a la densidad general (excepto para aquellos grupos con 3 o menos vértices). Si bien el sector productivo es el más numeroso, los tres sectores se 
encuentran vinculados con nodos puente entre cada sector. Lo cual facilita la difusión de información desde todos los ámbitos. La actividad económica "Programación informática, consultoría de informática y actividades conexas" es la más numerosa lo cual es consistente con el hecho de que es la actividad de la organización estudiada. Con respecto a los stakeholders se observan escasos contactos con clientes. A partir de una consulta con quien facilitó la red, indica que la mayoría de los clientes no registra un perfil en LinkedIn.

Características relacionales expresadas por miembros de la red LinkedIn. Para indagar sobre las propiedades relacionales se suministró un cuestionario $\mathrm{y}$ quince de los contactos respondieron. Puede observarse que los contactos no perciben que la red social tenga una influencia facilitadora en la transferencia y absorción de la información y el conocimiento (la moda de las respuestas corresponde con la apreciación "Muy poco"). En la fuerza de la relación se destaca la confianza mutua percibida entre los integrantes y la percepción de que las redes sociales que facilitan la colaboración y el conocimiento muto con otras organizaciones, contribuyen al éxito futuro de la empresa.

Figura 3 - Zuntini Soluciones Integrales. Red definida a partir de la red social profesional Linkedin. El grupo está definido en base al sector. El tamaño de los vértices se relaciona con el grado (el tamaño máximo se mapea con la media del indicador). La forma se relaciona con el stakeholder: Cliente (círculo), Cliente potencial (disco), Empleado (esfera), Gobierno (cuadrado), Proveedor (cuadrado sólido), Sociedad (diamante), Socios (diamante sólido).

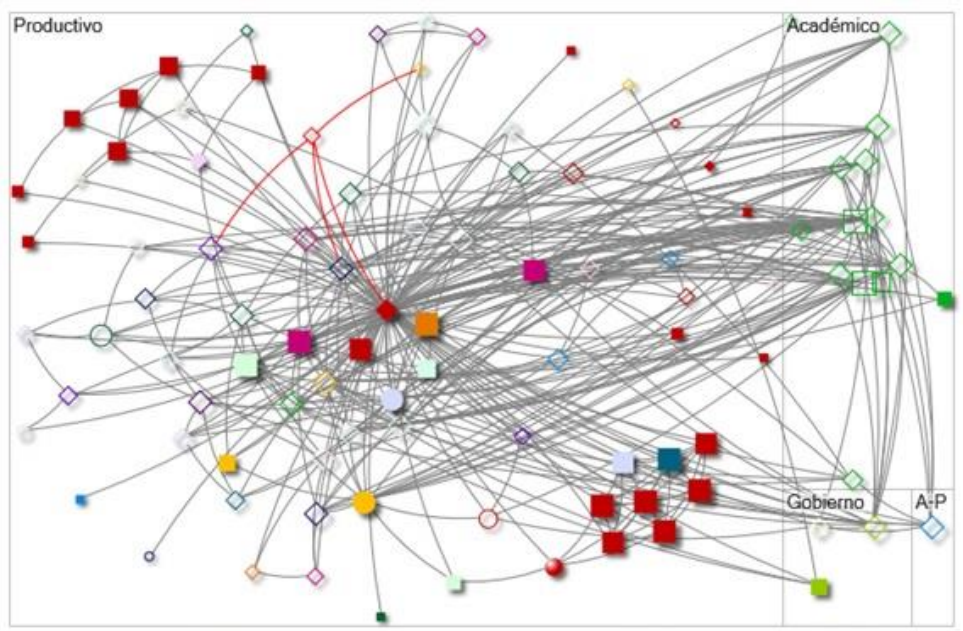

Fuente: elaboración propia utilizando el software NodeXL®. 
Figura 4 - Zuntini Soluciones Integrales. Red definida a partir de la red social profesional LinkedIn. El grupo está definido en base al stakeholder. El tamaño de los vértices se relaciona con el grado (el tamaño máximo se mapea con la media del indicador). Algoritmo Fruchterman Reingo.

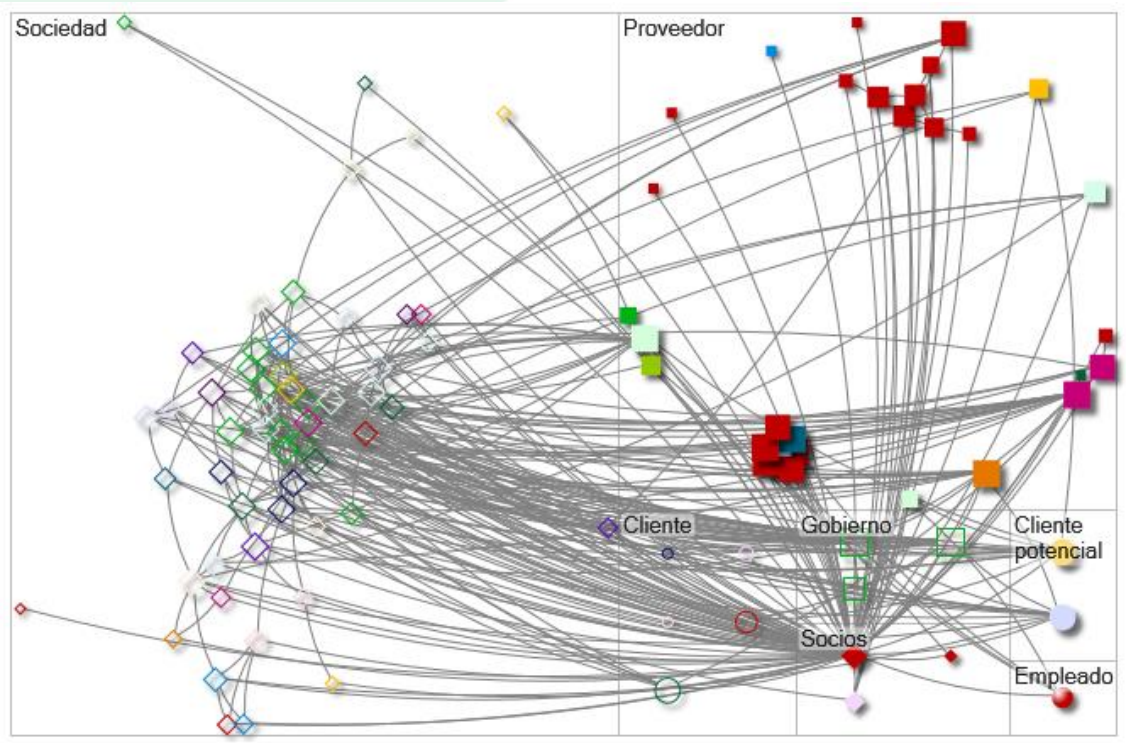

Fuente: elaboración propia utilizando el software NodeXL®.

Figura 5 - Zuntini Soluciones Integrales. Red definida a partir de la red social profesional Linkedin. El grupo está definido en base a la actividad económica. El tamaño de los vértices se relaciona con el grado (el tamaño máximo se mapea con la media del indicador). La forma se relaciona con el stakeholder. Algoritmo Fruchterman Reingo.

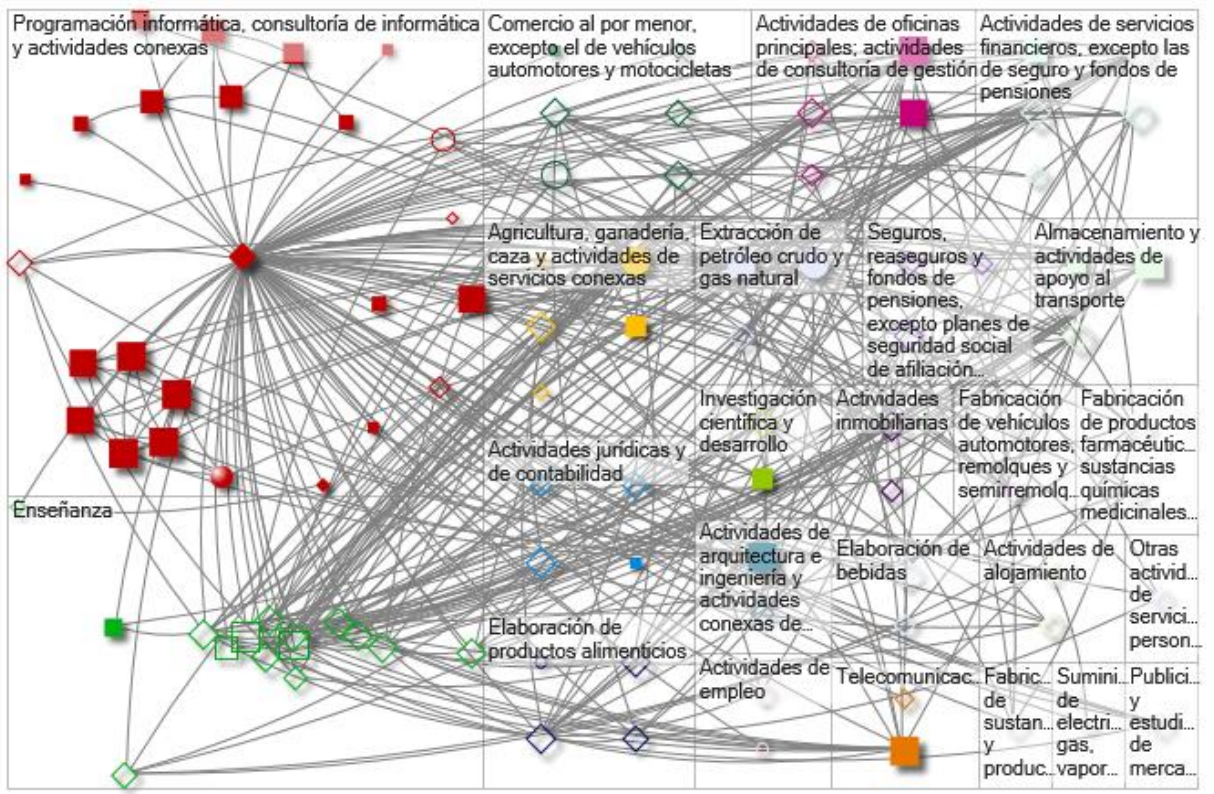

Fuente: elaboración propia utilizando el software NodeXL®.

\subsection{Prevención Salud}

\section{Descripción de la organización}

Se trata de una organización de origen nacional, fundada hace más de 20 años, dedicada al sector de servicios de salud actuando como aseguradora de riesgos de trabajo. Actualmente, su estructura global consiste en una casa matriz y diversas oficinas distribuidas en todo el territorio argentino. Ha extendido sus actividades en diversos países, convirtiéndose en una empresa internacional. Su misión es proteger la salud del personal de las empresas aseguradas en todo el país, con el 
compromiso de contribuir a la reducción del riesgo de los accidentes y enfermedades profesionales otorgando una atención eficiente y efectiva a los empleados afectados. Entre los clientes más importantes a los que brinda sus servicios se destacan aquellos pertenecientes al sector de los agronegocios, las industrias productoras de alimentos y las empresas de elaboración de sustancias químicas. Con respecto a la estructura de aprovisionamiento de los recursos, sus proveedores más significativos corresponden a organizaciones prestadoras de servicios de información, tecnologías y comunicación, consultoría, asesoría en coberturas y riesgos específicos según la actividad asegurada, organizaciones de reclutamiento para recursos humanos y profesionales de la salud. En relación a los competidores y rivales de la organización existen pocas firmas con presencia fuerte y larga permanencia en el mercado.

Facebook. Se importó la red correspondiente al período del 1 al 30 de junio de 2016 y se computaron los datos incluidos en la Tabla 10. Para el periodo analizado se observa una publicación diaria de posts los cuales corresponden en su mayoría a mensajes vinculados con noticias y consejos vinculados con el bienestar y la prevención de la salud. El resto de los posts se refieren promociones de productos, mensajes de clientes, y felicitaciones por el día del bioquímico, del libro, del padre, del donante de sangre y de la medicina preventiva.

LinkedIn. A partir de la lista de contactos se procesó la información (Figura 6). Debido a restricciones en el acceso a la red LinkedIn solo se obtuvo la lista de contactos de la red (es decir, no se pudo recuperar la red de grado 1.5). Se obtuvo la lista utilizando la herramienta Socilab. El 25\% de los contactos se desempeña en la actividad "Seguros, reaseguros y fondos de pensiones, excepto planes de seguridad social de afiliación obligatoria".

Figura 6 - Prevención Salud. Contactos clasificados de acuerdo al sector y stakeholder.

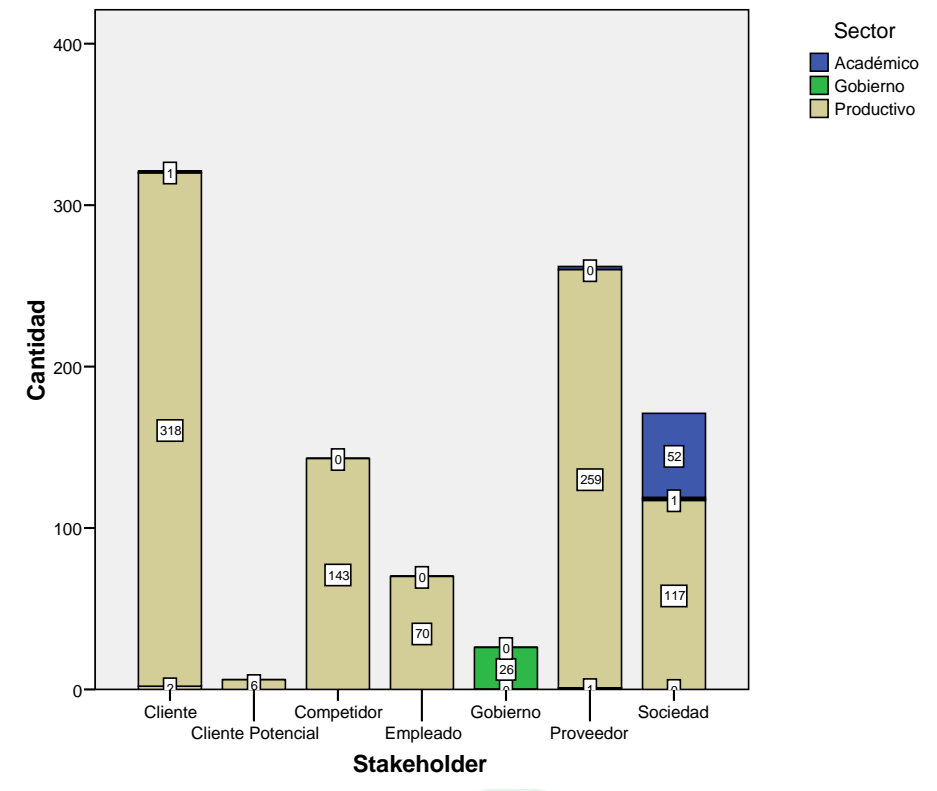

Fuente: elaboración propia a partir de los datos procesados. 
Tabla 10 - Descripción sintética de las Fan Page analizadas.

\begin{tabular}{|lcc|}
\hline \multicolumn{1}{|c|}{ Nombre de la Fan Page o grupo } & Zuntini* & Prevención Salud** \\
\hline Inicio & Sector Productivo & 2014 \\
Número de seguidores/miembros & 2014 & 290.296 \\
\hline Frecuencia de la actividad & 890 & Diaria \\
\hline Me gusta creados promedio & Semanal & 1,37 \\
Comentarios creados promedio & 2.167 & 1,36 \\
\hline Vértices & 0 & 1414 \\
\hline Arcos & 6 & 35896 \\
\hline Densidad & 4 & 0,017 \\
\hline
\end{tabular}

Referencias: * Período observado del 1/1/2016 al 31/6/2016. ** Período observado del 1/6/2016 al 31/6/2016. Fuente: elaboración propia.

\subsection{AF Equipamientos}

AF Equipamientos Industriales S.R.L. nace en 1999 en Bahía Blanca, Argentina, con el propósito de proveer equipos mecánicos, eléctricos y de instrumentación al mercado local y a la zona sur del país, brindando paralelamente un servicio de ingeniería y asistencia técnica. Con ya 15 años en el mercado, satisface los requerimientos del sector industrial nacional y latinoamericano y se ha consolidado como proveedora de servicios de ingeniería para la construcción y mantenimiento de plantas industriales. En la actualidad, su mercado abarca industrias químicas, petroquímicas, del petróleo y gas, siderúrgicas, farmacéuticas, mineras, pulpa y papel.
Linkedin. Tal como para el caso de Prevención Salud, solo se pudo obtener la lista de contactos. Se clasificó a cada contacto de acuerdo al sector, stakeholder (Figura 7) y la actividad económica. La actividad mayoritaria (19\%) corresponde con "Extracción de petróleo crudo y gas natural", siguiendo "Suministro de electricidad, gas, vapor y aire acondicionado" con el $7 \%$.

\subsection{Características relacionales de las redes sociales}

Se suministró el cuestionario a los contactos de cada uno de los tres casos. Además, para el caso de Zuntini Soluciones Integrales, 15 contactos respondieron el mismo.

Figura 7 - AF Equipamientos. Clasificación de los contactos en Linkedin de acuerdo al sector y al tipo de stakeholder con respecto a la organización en estudio.

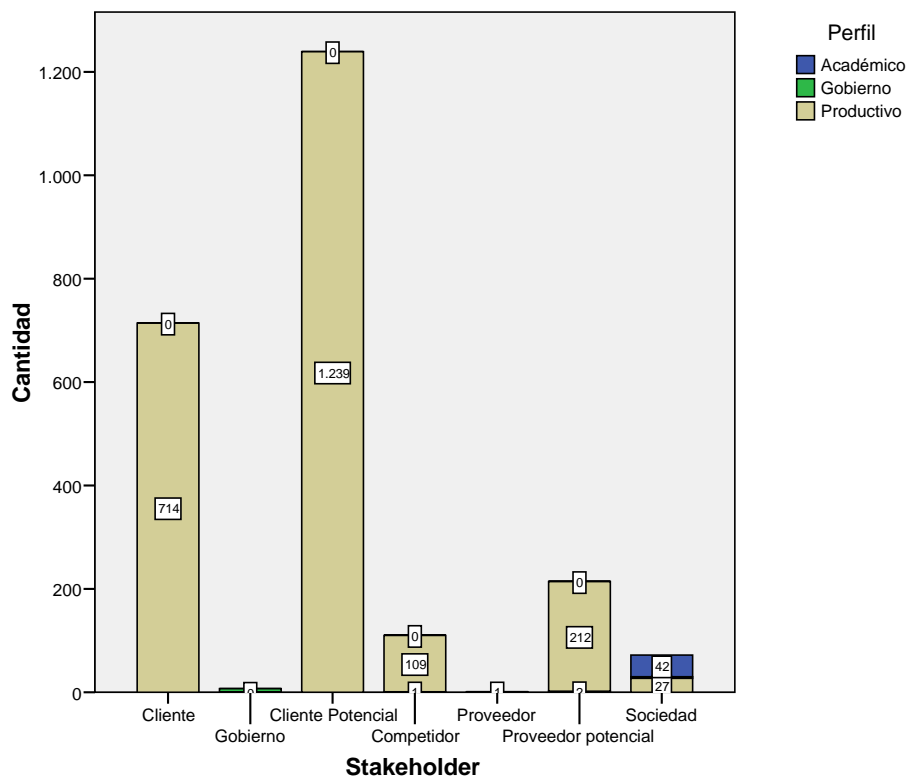

Fuente: elaboración propia a partir de los datos procesados. 
Tabla 11 - Descripción de las redes desarrolladas a partir de contactos en LinkedIn.

\begin{tabular}{|c|c|c|c|c|c|c|c|c|c|c|c|c|c|c|}
\hline \multirow[b]{2}{*}{ Organización } & \multicolumn{4}{|c|}{ Sectores } & \multicolumn{9}{|c|}{ Stakeholders } & \multirow[b]{2}{*}{ Еี } \\
\hline & 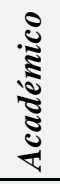 & $\frac{8}{5}$ & 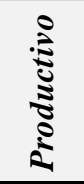 & $\stackrel{7}{\pi}$ & $\underset{:}{\stackrel{\Xi}{\Xi}}$ & 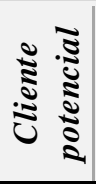 & 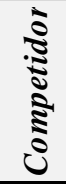 & 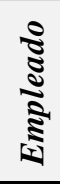 & $\frac{\mathfrak{5}}{3}$ & 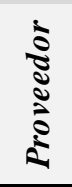 & 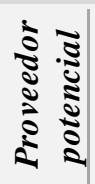 & 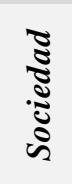 & 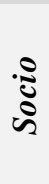 & \\
\hline Zuntini & 14 & 3 & 81 & 1 & 5 & 2 & - & 1 & - & 31 & - & 58 & 2 & 99 \\
\hline Prevención Salud & 55 & 27 & 917 & - & 321 & 6 & 143 & 70 & 26 & 262 & - & 171 & - & 999 \\
\hline $\begin{array}{c}\mathrm{AF} \\
\text { Equipamientos }\end{array}$ & 42 & 10 & 2305 & - & 714 & 1239 & 110 & - & 7 & 1 & 214 & 72 & & 2357 \\
\hline
\end{tabular}

Fuente: elaboración propia a partir de los datos procesados.

Los resultados de los cuestionarios sobre las características relacionales reflejan que los usuarios no tienen una percepción de que la transferencia y absorción de información y conocimiento a través de la red social virtual sea muy importante. La moda para esas preguntas es 1 y 2 , lo cual se corresponde con que los usuarios perciben que se enriquecen muy poco con la red. Con respecto a la fuerza de la relación, la cantidad de temas promedio sobre los cuales los usuarios se benefician a partir de la red es 1,39 sobre un total de seis temas listados en el cuestionario. La moda de las respuestas vinculada con la afirmación sobre la existencia de honestidad y confianza entre los contactos es tres (el valor intermedio en la escala utilizada de cinco puntos). El segundo valor más frecuente es cuatro, lo cual refleja una tendencia a estar de acuerdo con la afirmación. Además, los usuarios son neutros con una tendencia positiva con respecto a la percepción de que las redes sociales virtuales contribuyen al éxito de la organización. Con respecto al número de membrecías en diferentes tipos de redes se observan dos grupos de respuestas: aquellos que registran ninguna o una membrecía; los que registran cuatro o cinco, y quienes informan nueve a doce. Todas las respuestas con similares tanto para los tres casos bajo estudio como para los contactos incluidos en la red profesional del caso Zuntini Soluciones Integrales. Entonces, si bien las interpretaciones anteriores se generalizan para todos los casos aunque cabe observar tres contactos de Zuntini Soluciones Integrales cuyas puntuaciones para las preguntas vinculadas con el flujo de información y fuerza de la relación superan ampliamente los valores para la moda. Finalmente, con respecto al desempeño general de la organización en los últimos tres años, Prevención Salud y AF Equipamientos han indicado que la participación en el mercado, las ventas, la utilidad sobre ventas, la productividad y el retorno sobre la inversión se aumentaron. Zuntini Soluciones Integrales coincide en las respuestas excepto para ventas, utilidad sobre ventas y retorno sobre la inversión, para los cuales indica que se mantuvieron.

\section{CONCLUSIONES}

La principal contribución del trabajo es el análisis de la utilización de redes sociales virtuales en organizaciones tomando como fuente de datos primaria las redes sociales (a diferencia de otras investigaciones que realizan encuestas). El análisis de las redes permitió describir qué sectores (académico, productivo y gubernamental) se encuentran conectados en la red. Asimismo, la clasificación de los miembros desde una perspectiva de stakeholders permite explorar si la estructura de la red propicia el intercambio de información y conocimiento entre los mismos. El procesamiento de la red también ilustra las actividades económicas y países vinculados a los miembros de la red virtual. De esta forma, se obtienen indicadores directos sobre la utilización de la red. Al mismo tiempo, dado que las conexión no constituyen una condición suficiente para que exista un intercambio de información y conocimiento se indagó utilizando un cuestionario como instrumento sobre aspectos relacionales que no pueden inferirse a partir del grafo de una red.

Analizando globalmente las características de cada red profesional estudiada y realizando una observación comparativa, surge que la organización "Prevención Salud" posee una mayor vinculación con actores del sector "Académico" y "Gobierno" a diferencia de las restantes organizaciones. En cuanto a las conexiones establecidas de las redes analizadas para el sector "Productivo", la organización "AF Equipamientos" presenta una significativa cuantía de relaciones con actores del mencionado sector, sucediéndole en los guarismos "Prevención Salud". Es destacable que no hay evidencia de vinculación entre el sector "Académico-Productivo" en ninguna de las redes analizadas para cada organización bajo estudio. Esto demuestra un vacío estratégico ante las posibilidades potenciales que podrían surgir de esta conexión, traducidas en ventajas competitivas para cada organización.

Desde el punto de vista comparativo de las vinculaciones con los stakeholders, "Prevención Salud" tiene la mayor participación relativa $(32,13 \%)$ de sus 
conexiones con Clientes, seguido por los vínculos con sus Proveedores $(26,23 \%)$, la Sociedad $(17,12 \%)$ y los Competidores $(14,31 \%)$. Los restantes sectores no tienen incidencia significativa en los lazos generados por la red de la firma. En la red de "Zuntini Soluciones Integrales" predominan los actores del sector Sociedad $(59,18 \%)$ y Proveedores (31,63\%). Asimismo, raramente se muestran vinculaciones con Clientes $(5,10 \%)$. "AF Equipamientos" focaliza sus relaciones con actores de la red que sean Clientes Potenciales (representan el 52,57\% de sus conexiones totales) y Clientes actuales (30,29\%). Seguidamente se mantienen conexiones con Potenciales Proveedores lo que puede resultar importante en el plano estratégico de abastecimiento de recursos productivos. Como se expone en el análisis, los restantes sectores intervinientes no alcanzan guarismos significativos. Cabe recordar que la existencia de contactos no es suficiente para inferir que exista una comunicación entre los actores.

A modo de conclusión sobre los casos, se observa que "Prevención Salud" refleja un accionar eficaz de la red virtual para transmitir información con los clientes. Esto se fundamenta en la cantidad de vínculos en la red profesional y los datos que surgen del perfil de la Fan Page. Para el caso de "Zuntini Soluciones Integrales" se observa que se utiliza la Fan Page como medio de interacción con clientes. Los contactos que surgen de la red profesional no representan a este último sector, pero esto se debe a que pocos clientes registran un perfil en LinkedIn. El caso de "AF Equipamientos" destaca por la cantidad y diversidad de contactos profesionales aunque el cuestionario no evidencia un convencimiento sobre la influencia de la red en la transmisión y absorción de información y conocimiento.

Las redes generalistas como Facebook resultan adecuadas para facilitar la adquisición y la retención de clientes potenciales. Tanto "Prevención Salud" como "Zuntini Soluciones Integrales" reflejan un accionar proactivo para el aprovechamiento de las Fan Page de Facebook para fortalecer los lazos con clientes y clientes potenciales.

Finalmente, si bien para las PyMES, la participación en una red social puede otorgar acceso a recursos y capacidades, y a partir del cuestionario suministrado a algunos contactos se advierte una percepción neutra con una tendencia positiva con respecto a la percepción de que las redes sociales virtuales contribuyen al éxito de la organización. Asimismo, los resultados del cuestionario indican que los usuarios no tienen una percepción de que la transferencia y absorción de información y conocimiento a través de la red social virtual sea muy importante. Esto también evidencia una oportunidad no aprovechada de convertir a la red en una fuente difusión y absorción de información y conocimiento tanto para los stakeholders internos como externos de una organización.

\section{REFERENCIAS}

Agrawal, D., Budak, C., \& El Abbadi, A. (2011). Information diffusion in social networks: Observing and affecting what society cares about. En Intl. Conf. on Information and Knowledge Management.

Ahlemann, F., El Arbi, F., Kaiser, M., \& Heck, A. (2013). A process framework for theoretically grounded prescriptive research in the project management field. International Journal of Project Management, 31, 43-56.

Aragõn, P., Kappler, K. E., Kaltenbrunner, A., Laniado, D., \& Volkovich, Y. (2013). Communication dynamics in twitter during political campaigns: The case of the 2011 spanish national election. Policy and Internet, 5(2), 183-206.

Archambault, A., \& Grudin, J. (2012). A Longitudinal Study of Facebook, Linkedin, and Twitter Use. Proceedings of the SIGCHI Conference on Human Factors in Computing Systems (págs. 2741-2750). Austin: ACM.

Argote, 1., \& Ingram, P. (2000). Knowledge Transfer: A Basis for Competitive Advantage in Firms. Organizational behavior and Human Decision Processes, 82(1), 150-169.

Chelmis, C., \& Prasanna, V. K. (2013). The role of organization hierarchy in technology adoption at the workplace. Proc. of the 2013 IEEE/ACM International Conference on Advances in Social Networks Analysis and Mining, ASONAM 2013, 8-15.

Chelmis, C., Srivastava, A., \& Prasanna, V. K. (2014). Computational models of technology adoption at the workplace. Social Network Analysis and Mining, 4(1), 1-18.

Chuang, S. (2004). A resource-based perspective on knowledge management capability and competitive advantage: an empirical investigation. Expert Systems with Applications, 27, 459-465.

Conti, M., Das, S., Bisdikian, M., Kumar, M., Ni, L., Passarella, A., . . . Zambonelli, F. (2012). Looking ahead in pervasive computing: Challenges and opportunities in the era of cyberphysical convergence. Pervasive and Mobile Computing, $8(1), 2-21$.

Crowne, K. A., Goeke, R. J., \& Shoemaker, M. (2015). Enhancing international assignees' performance with online social networks. Journal of Global Mobility, 3(4), 397-417. 
Darr, E., \& Kurtzberg, T. (2000). An Investigation of Partner Similarity Dimensions on Knowledge Transfer. Organizational Behavior and Human Decision Processes, 82(1), 28-44.

Edwards, G. (2010). Mixed-Method Approaches to Social Network Analysis. ESRC National Centre for Research Methods.

Ellison, N., Gibbs, J., \& Weber, M. (2015). The use of enterprise social networks sites for lnowledge sharing in distributed organization: the roles of organizational affordances. American behavioral Scientist, 29(1), 103-123.

Estevez, E., \& Janowski, T. (2013). Electronic Governance for Sustainable Development Conceptual Framework and State of Research. Government Information Quarterly, 30(1), S94S109.

Etzkowitz, H. (2003). Innovation in Innovation: The Triple Helix of University-Industry-Government Relations. Social Science Information, 42(3), 293337.

Etzkowitz, H., \& Leydesddorff, L. (1995). The Triple Helix -University-Industry-Government Relations: A Laboratory for Knowledge Based Economics Development. EASST Review, 14(1), 11-19.

Fritsch, M., \& Kauffeld-Monz, M. (2010). The impact of network structure on oknowledge transfer: an application of social network analysis in the context of regionanl innovation networks. The Annals of Regional Science, 44, 21-38.

Fu, X., Passarella, A., Quercia, D., Sala, A., \& Strufe, T. (2016). Online Social Networks. Computer Communications, 73(Part B), 163-166.

Fuerlinger, G., Fandl, U., \& Funke, T. (2015). The role of the state in the entrepreneurship ecosystem. Insights from Germany. Triple Helix, 2(3), 1-26.

Garg, R., Smith, M. D., \& Telang, R. (2011). Measuring information diffusion in an online community. Journal of Management Information Systems, 28(2), 11-37.

Gilsing, V., \& Duysters, G. (2008). Understanding novelty creation in exploration networks -Structural and relational embeddedness jointly considered. technovation, 28, 693-708.

Granovetter, M. (1973). The strength if weak ties. American Journal of Sociology, 78, 1360-1389.
Grant, R. (1996). Toward a knowledge-based theory of the firm. Strategic Management Journal, 32(4), 109-122.

Hanneman, R., \& Riddle, M. (2005). Introduction to social network methods. Riverside: University of California.

Haythornthwaite, C. (1996). Social Network analysis: An Approach and Technique for the Study of Information Exchange. Library \& Information Science Research, 18(4), 323-342.

He, W., \& Wang, F. (2016). A process-based framework of using social media to support innovation process. Information Technology Management, 17(123).

Heimbach, I., \& Hinz, O. (2016). The impact of content sentiment and emotionality on content virality. International Journal of Research in Marketing, 33(3), 695-701.

Jiang, B., Wang, L., Yang, C., Peng, S., \& Li, R. (2014). Modeling the information propagation in an email communication network using an agent-based approach. Proc. GECCO 2014, 1007-1014.

Korzynski, P. (2015). Online networking and employee engagement: What current leaders do? Journal of Management Psychology, 30, 582-596.

Levy, M., Hadar, I., Te'eni, D., Unkelos-Shpigel, N., Sherman, S., \& Harel, N. (2016). Social networking in an academic conference context: Insights from a case study. Information Technology and People, 29(1), 51-68.

Li, D., Zhang, Y., Xu, Z., Chu, D., \& Li, S. (2016). Exploiting information diffusion feature for link prediction in sina weibo. Scientific Reports, 6 .

Liang, Q., Liao, X., \& Liu, J. (2017). A social tiesbased approach for group decision-making problems with incomplete additive preference relations. Knowledge-Based Systems, 119, 68-86.

Lim, S. -., Kim, S. -., Park, S., \& Yoon, S. -. (2011). Determining diffusion power users in a blogosphere. Information, 14(8), 2635-2653.

Lin, C., Xie, R., Guan, X., Li, L., \& Li, T. (2014). Personalized news recommendation via implicit social experts. Information Sciences, 254, 1-18.

Lin, C., Xie, R., Li, L., Huang, Z., \& Li, T. (2012). PRemiSE: Personalized news recommendation via implicit social experts. ACM International Conference Proceeding Series, 1607-1611. 
Lipizzi, C., Iandoli, L., \& Marquez, J. E. R. (2016). Combining structure, content and meaning in online social networks: The analysis of public's early reaction in social media to newly launched movies. Technological Forecasting and Social Change, 109, 35-49.

Luo, S., Du, Y., Liu, P., Xuan, Z., \& Wang, Y. (2015). A study on coevolutionary dynamics of knowledge diffusion and social network structure. Expert Systems with Applications, 42, 3619-3633.

Manetti, G., \& Bellucci, M. (2016). The use of social media for engaging stakeholders in sustainability reporting. Accounting, Auditing \& Accountability Journal, 29(6), 985-1011.

Mozafari, N., \& Hamzeh, A. (2015). An enriched social behavioural information diffusion model in social networks. Journal of Information Science, 41(3), 273-283.

Naciones Unidas. (2009). Clasificación Industrial Internacional Uniforme de todas las actividades económicas (CIIU). Revisión 4. Dpto. Asuntos Económicos y Sociales. Nueva York: Naciones Unidas.

Narayanan, M., Asur, S., Nair, A., Rao, S., Kaushik, A., Mehta, D., . . . Lalwani, R. (2012). Social Media and Business. Vikalpa: The journal for Decision Makers, 37(4), 69-111.

Palacios-Marqués, D., Popa, S., \& Alguacil Mari, M. (2016). The effect of online social networks and competency-based management on innovation capability. Journal of Knowledge Management, 20(3), 499-511.

Sankar, M. V., \& Ravindran, B. (2015). Parallelization of game theoretic centrality algorithms. Sadhana Academy Proceedings in Engineering Sciences, 40(6), 1821-1843

Shin, S., Lee, K., \& Hall, D. (2014). Exploring Facebook Users' Continuous Visiting Behaviors: Conceptual Incorporation of Facebook User Perceptions toward Companies Facebook Fan Page Usage. Twentieth Americas Conference on Information Systems. Savannah.

Skeels, M., \& Grudin, J. (2009). When social networks cross boundaries: a case study of workplace use of Facebook and Linkedin. Proc. of the ACM 2009 Intl Conf. on Supporting Group Work (p. 95-104). Florida: ACM.

Smith, A. D. (2013). Online social networking and office environmental factors that affect worker productivity. International Journal of Procurement Management, 6(5), 578-608.

Subires Mancera, M., \& Olmedo Salar, S. (2013). Universidad, sociedad y networking: perspectivas ante el uso de las redes sociales de perfil académico profesional. Estudios sobre el Mensaje Periodístico, 19, 1037-1047.

Roy, S. D., \& Zeng, W. (2015). Social multimedia signals: A signal processing approach to social network phenomena. Cham: Springer International Publishing.

Tang, X., Miao, Q., Quan, Y., Tang, J., \& Deng, K. (2015). Predicting individual retweet behavior by user similarity: A multi-task learning approach. Knowledge-Based Systems, 89, 681-688.

Van Alstyne, M., \& Brynjolfsson, E. (2005). Global village or CyberBalkans: Modeling and measuring the integration of electronic communities. Management Science, 51(6), 851-868.

Wadhwa, P., \& Bhatia, M. P. S. (2015). Measuring radicalization in online social networks using markov chains. Journal of Applied Security Research, 10(1), 23-47.

Wang, Q., Lin, Z., Jin, Y., Cheng, S., \& Yang, T. (2015). ESIS: Emotion-based spreader-ignorantstifler model for information diffusion. KnowledgeBased Systems, 81, 46-55.

Wu, D., Li, C., \& Lau, R. Y. K. (2015). Topic based information diffusion prediction model with external trends. 12th IEEE International Conference on E-Business Engineering, ICEBE 2015, 29-36.

Yang, C., Jiang, B., \& Wang, L. (2016). Mining and modeling the information propagation in an email communication network. Proc. HCC 2016, Colombo, Sri Lanka.

Yin, R. (1994). Case Study Research: Design and Methods. Thous and Oaks: Sage Publications.

Zaglia, M., Waiguny, M., Abfalter, D., \& Müller, J. (2015). The influence of online social networks on performance of small and medium enterprises: An empirical investigation of the online business to business network XING. Int. J. Entrepreneurial Venturing, 7(1), 1-23.

Zhang, J. (2015). Visual analytics of user influence and location-based social networks. En Transparency in social media: Tools, methods and algorithms for mediating online interactions. Springer International Publishing. 
La Influencia de las Redes Sociales Virtuales en la Difusión de Información y Conocimiento: Estudio de PyMES

Zhang, J., Yu, P., Lv, Y., \& Zhan, Q. (2016). Information Diffusion at Workplace. CIKM, 1673-

1682, Indianapolis: ACM. 